\title{
Evaluating Insulin/Glucose Ratio Using Breakfast of Calorie Restriction Meal for Type 2 Diabetes Mellitus
}

\author{
Ebe $\mathrm{K}^{1,2}$, Bando $\mathrm{H}^{2,3^{*}}$, Muneta $\mathrm{T}^{2,4}$, Bando $\mathrm{M}^{5}$, Yonei $\mathrm{Y}^{6}$ \\ ${ }^{\text {I}}$ Takao Hospital, Kyoto, Japan \\ ${ }^{2}$ Japan Low Carbohydrate Diet Promotion Association, Kyoto, Japan \\ ${ }^{3}$ Tokushima University / Medical Research, Tokushima, Japan \\ ${ }^{4}$ Muneta Maternity Clinic, Chiba, Japan \\ ${ }^{5}$ Department of Nutrition and Metabolism, Institute of Biomedical Sciences, Tokushima University Graduate School, \\ Tokushima, Japan \\ ${ }^{6}$ Anti-Aging Medical Research Center, Graduate School of Life and Medical Sciences, Doshisha University, Kyoto, \\ Japan
}

*Correspondence: Hiroshi Bando, MD, PhD, FACP

Received on 04 December 2018; Accepted on 25 January 2019; Published on 01 February 2019

Copyright $(92019$ Ebe $\mathrm{K}$ et al. This is an open access article and is distributed under the Creative Commons Attribution License, which permits unrestricted use, distribution, and reproduction in any medium, provided the original work is properly cited.

\begin{abstract}
Background: Type 2 diabetes mellitus (T2DM) has been a medical and social problem worldwide. For nutritional therapy, Low Carbohydrate Diet (LCD) and Calorie restriction (CR) have discussed for long, where authors continued clinical research. Subjects and Methods: Subjects were 68 patients with T2DM with 62.1 years on average. Methods included i) standard CR was provided a day with $1400 \mathrm{kcal}$ and $60 \%$ of carbohydrate, ii) measurement of daily profile of blood glucose, iii) insulinogenic index (IGI) (0-30 mins) exam for breakfast including $70 \mathrm{~g}$ of carbohydrate, iv) calculation of IGI by delta and Area Under the Curves(AUC).

Results: Basal data revealed that HbAlc 7.9\%, fasting glucose $163 \mathrm{mg} / \mathrm{dL}$, average glucose in a day $210 \mathrm{mg} / \mathrm{dL}$, $M$ value 117 in the median. Increment of glucose/IRI was $48.0 \mathrm{mg} / \mathrm{dL} / 8.6$ $\mu U / m L$, respectively. Delta or AUC ratio of IGI was 0.14[0.08-0.26] and 3.3[2.5-5.2], respectively. There were significant correlations between $M$ value and Delta or AUC ratio, with a higher coefficient in the latter.

Discussion and Conclusion: IGI study in Meal Tolerance Test (MTT) would be useful for pancreas function evaluation. AUC ratio method has superiority than Delta ratio with higher correlation coefficient. Current results could be the fundamental data for the related range of research, and further development will be expected.
\end{abstract}

Keywords: area under the curves (AUC), insulinogenic index (IGI), type 2 diabetes mellitus (T2DM), morbus value (M value), delta ratio of IGI, AUC ratio of IGI

Abbreviation: AUC: Area Under the Curve; IGI: Insulinogenic Index; T2DM: Type 2 Diabetes Mellitus; M value: Morbus value; IRI: Imuunoreactive Insulin 


\section{Introduction}

In recent years, various nutrition and metabolic problems have been observed in developing and developed countries. One of the crucial diseases is Metabolic syndrome (Met-S). It includes obesity and diabetes, hypertension, hyperlipidemia, which was syndrome $\mathrm{X}$ in 1980-90s [1]. After the accumulation of lots of research, Met-S was proved to cause several arteriosclerotic diseases through insulin resistance mechanism [2,3]. In relation to these pathophysiological situations, many influencing factors are present such as meal, exercise, age, lifestyle and so on.

As to Met-S, diabetic predisposition can be involved in basically influencing the impaired function such as insulin resistance. The prevalence of diabetes has been also increasing worldwide, and its problems include macroangiopathic and micro-angiopathic complications [4,5]. For better management of diabetes, there has been a recent proposal for changes of comments from medical societies, including American Diabetes Association (ADA) and American College of Physicians (ACP) [6,7]. The controversy has been in discussion about mainly the ideal HbA1c level in clinically various conditions. On the other hand, International Diabetes Federation (IDF) has proposed the standards of medical care in diabetes [8]. Among lots of recommendations, it is the intake and counting amount for carbohydrate that has been emphasized [8].

The problem of carbohydrate intake in diabetes or Met-S has been widely-known in nutritional therapy. The discussion has been continued concerning the comparison of mainly two diet methods. They are usually compared between Low Carbohydrate Diet (LCD) and Calorie restriction (CR) [9]. The difference of the concept is that LCD means the decreased intake of carbohydrate as meals, while CR means the decreased intake of fat leading to less calorie restriction a day $[10,11]$.

There are many papers compared with LCD and CR [12]. Recently, the results of "The Prospective Urban Rural Epidemiology (PURE) study" was reported. It covered the investigation from 140 thousand subjects from 18 countries in the world [13]. The results showed that higher carbohydrate intake was observed associated with an increased risk of HR 1.28 of total mortality. Consequently, taking lower carbohydrate seemed to be recommended for everyone to lead a better life [14].

From various data, IDF proposed the Standards of Medical Care in Diabetes [15]. In this guideline, the important points seemed to be monitoring the amount of carbohydrate intake, carbohydrate counting, and experience-based estimation. Furthermore, continuing evaluation and study of eating patterns and macronutrient distribution would have beneficial results for achieving better glycemic control in the future.

Concerning LCD, it was started by Bernstein who was a physician with type 1 diabetes mellitus (T1DM) [16]. After that, LCD became rather prevalent [12]. On contrast in Japan, authors and colleagues have initiated LCD and developed medically and socially with Japan LCD promotion association, and have given adequate therapy for many diabetic patients with successful treatment [17]. Furthermore, we have continued LCD research related to lipid metabolism, renal function, elevated ketone bodies and three types of LCD formula which are petit, standard and super LCD [18-20].

Through our clinical research on CR and LCD, we have provided formula diet of CR and LCD and performed the examination. We have proposed a meal tolerance test (MTT) and investigated insulinogenic index (IGI) using breakfast including $70 \mathrm{~g}$ of carbohydrate, similar to $75 \mathrm{~g}$ oral glucose tolerance test (75g OGTT) [21].

In this study, we have developed this research, including the comparison of the analysis methods between delta (increment) and area under the curves (AUC).

\section{Materials and Methods}

In the current study, subjects enrolled were 68 patients with type 2 diabetes mellitus (T2DM). The subjects enrolled were 68 patients with type 2 diabetes mellitus. Male and female were 40 and 28 , respectively. The average age was $62.1 \pm 10.9$ years old (mean \pm standard deviation), and 65 [57-69] years old (median and quartile of 25\%-75\%). 
For the standard research protocol method for T2DM, subjects were admitted to the hospital. With regard to the condition of the subjects, we selected the patients with several necessary points as follows: 1) the diagnosis is T2DM, 2) another diagnosis such as T1DM were excluded, 3) subjects on hemodialysis or with heavy chronic renal failure or heavy liver disease were excluded, 4) subjects on insulin therapy were excluded, 5) patients with its body mass index (BMI) 35 and more than 35 were excluded, 6) patients with its immunoreactive insulin (IRI) level was 5 and more than $5 \mu \mathrm{U} / \mathrm{mL}$ were excluded.

This protocol is called "Educational admission for learning diabetes" for two weeks. There are some purposes for this understanding and treating experience for diabetes. They are i) to understand the fundamental correct information about diabetes, ii) to experience the meal of Calorie Restriction (CR) meal on day 1 and 2, and the meal of LCD from day 3 to day 14, iii) to evaluate general medical condition, besides diabetes mellitus, iv) to improve glucose variability remarkably within two weeks. Consequently, this protocol program would be for new diabetic patients usually after diagnosed as diabetes.

Methods for this study were along to our standard diabetic examination using the nutritional treatment for CR and LCD. In this study, the detailed content of the protocol were:

- $\quad$ Subjects were to have the standard meals, in which CR is served in on day 1,2, and LCD is served after day 3. It is along to our protocol method for clinical diabetes research. However, in the current study, we have analyzed the data from day 2 only.

- On day 2, we draw blood samples after overnight fasting in order to check basal biomarkers related to diabetes treatment and research. They are HbA1c, glucose, IRI, lipids, renal function, and complete blood count and so on.

- $\quad$ Subjects were provided breakfast in the morning on day 2, which was the standard formula for a test meal. It is standard Calorie Restriction (CR) meal, which is along the guideline from the Japan Diabetes Society (JDS) on diabetic nutritional manual [22]. The standard type of calorie restriction (CR) meal has 1400 $\mathrm{kcal} /$ day, with $60 \%$ of carbohydrate for 3 meals with breakfast, lunch and supper. The $60 \%$ of $1400 \mathrm{kcal}$ would be $840 \mathrm{kcal}$ for carbohydrate. Carbohydrate $1 \mathrm{~g}$ has $4 \mathrm{kcal}$, then $840 / 4=210 \mathrm{~g}$ of carbohydrate is taken a day. There are three meals per day, breakfast, lunch, and supper, then breakfast includes $70 \mathrm{~g}$ of carbohydrate. This is one of the meal tolerance test (MTT), using breakfast of calorie restriction (CR) meal.

- $\quad$ On pre and post 30 mins of breakfast, a blood sample was drawn. Subjects were told to keep still on sitting on the chair. The values of blood glucose, IRI, c-peptide were measured on 0 and 30 mins of the breakfast.

- $\quad$ For the daily profile of blood glucose, blood was drawn during the day. The clock time were 08, 10, 12, 14, 17,19 , and $22 \mathrm{~h}$. According to the results of blood glucose 7 times a day, we calculated average blood glucose value and also Morbus (M) value.

- During the investigation for two weeks, the patients with type 2 diabetes mellitus have been followed without oral anti-diabetic agents or any other medicine. The reason is that they are always observed carefully with frequent measurement of blood glucose and that their glucose variability for CR meal and LCD meal are evaluated.

- Regarding the measurement of blood glucose and HbA1c, the measurement kits were in the following. The level of blood glucose was measured using the Glu test Mint by SKK Sanwa Co. Ltd., Nagoya, Japan. On the other hand, the level of HbA1c was measured using the Accu-Chek Aviva Nano (Roche) by Roche Diagnostics, Tokyo, Japan.

- As to the laboratory measurement for the biochemistry of the blood test including liver function, renal function and lipid metabolism, clinical testing services of the FALCO Biosystems Ltd. Group were applied. These systems were automatically measured for the concentrations and activities of lots of biomarkers. Blood samples were drawn in fasting states after the overnight fast, in the measurement of glucose, immunoreactive insulin (IRI), triglyceride, HDL-cholesterol, LDL-cholesterol, and other biomarkers.

\section{Responses of glucose and insulin}

The research of test meal for the subjects would be meal tolerance test (MTT). As to the responses of glucose and IRI on 0 and 30 mins for the MTT, two calculation methods of Insulinogenic index (IGI) were performed.

As a reference to 75g OGTT, IGI has been familiar in order to suppose the ability of secretion of insulin from the pancreas. Its formula has been the delta (increment) of IRI (0-30 mins)/delta (increment) of blood glucose (0-30 mins). In this paper, we applied the same IGI for carbohydrate 70g. It is called the Delta Ratio of IGI for Carbo70. 
Another calculation was performed and investigated. For the usage of a square of the AUC, the responses of glucose and insulin were described. In comparison with the square area size, the ratio of IRI/glucose ratio was measured. It is called the AUC Ratio of IGI for Carbo70.

In summary of two methods, the formula would be in the following. Delta Ratio of IGI for Carbo70 is (IRI at 30 mins-

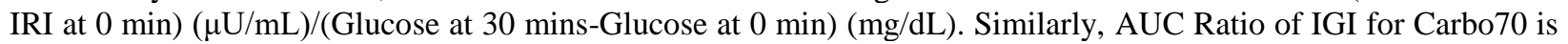
(AUC of IRI for 0-30 mins) ( $\mu \mathrm{U} / \mathrm{mL} \times \mathrm{h}) /($ AUC of glucose for $0-30 \mathrm{mins})(\mathrm{mg} / \mathrm{dL} \times \mathrm{h})$.

\section{Daily profile of glucose}

Regarding the daily profile of blood glucose, blood samples were drawn 7 times on day 2. The clock time as 08,10 , $12,14,17,19,22 \mathrm{~h}$. From the previous papers, there observed the similar and compatible results in comparison with 7-times sampling and 20 times sampling [23,24]. It has also the compatible results compared with the data from the continuous glucose monitoring (CGM) [23,24].

\section{Morbus value}

Morbus value has been introduced and known as one of the useful biomarkers for glucose variability [25,26]. It indicates two pathophysiological aspects, which are the average blood glucose and also the mean amplitude of glycemic excursions (MAGE).

In other words, $\mathrm{M}$ value can suggest the degree of hyperglycemia and also the degree of high fluctuation of blood glucose in a day. The calculation for the $M$ value has been used by mathematical way of logarithmic transformation. It suggests the deviation of glucose variability from ideal glucose average and swinging of daily profile [26,27].

There is the formula to calculate the $M$ value as follows: i) the basic equation is that $M=M B S+M W$, and $M$ value is the total of MBS and MW. ii) MW is calculated as (maximum blood glucose-minimum glucose)/20. iii) MBS is the mean of MBSBS. As these are summarized, MBSBS is the individual M-value for each blood glucose, calculated as (absolute value of $[10 \times \log ($ blood glucose level/120)])3 [25-27].

For clinical evaluation of $\mathrm{M}$ value, the usual standard levels are in the following: less than 180 would be normal range, 180-320 would be borderline, and more than 320 would be abnormal.

\section{Statistical analysis}

Regarding the statistical analysis in the current study, obtained data in this study were shown using the mean and standard deviation. Furthermore, they are shown using the median and quartile for $25 \%$ and $75 \%$ in some biomarkers where there is a necessary situation.

The latter are described as median and (25\%-75\%) values in the tables. As to the calculation of statistical analysis, Spearman test has been utilized and the correlation coefficients were obtained [28]. The sample size of this study would be adequate for the investigation of statistical analysis by Spearman test.

\section{Ethical standard}

The current study was performed with the compliance of the ethical principles which were from the Declaration of Helsinki, and from 2004 General Assembly Tokyo, Japan. Furthermore, they were performed according to the Personal Information Protection Law and in reference to "Standards for the Implementation of Clinical Trials (GCP), which are from an ordinance of the Ministry of Health, Labour and Welfare No. 28 of March 27, 1997. In addition to these principles, the "Ethical Guidelines for Epidemiology Research" were presented and applied by the Ministry of Education, Culture, Sports, Science and Technology and the Ministry of Health, Labour and Welfare.

Authors have continued an ethical committee including some professionals such as a doctor, registered nurse, pharmacist and other experts in the legal specialty. Authors and colleagues have discussed and resulted that this investigation has been valid and agreed with all members.

From all subjects, we have taken informed consents and written paper agreements. This study has been registered by National University Hospital Council of Japan (ID: \#R000031211). 


\section{Results}

\section{Basal data}

Basal data taken from the morning on Day 2 were shown (Table 1). The average age was 62.1 years old, and the average $\mathrm{HbAlc}$ was $7.9 \%$ on average. Fasting blood glucose was $163 \mathrm{mg} / \mathrm{dL}$, and average blood glucose in a day was $210 \mathrm{mg} / \mathrm{dL}$ on average. Median levels of M value, HOMA-R and HOMA- $\beta$ were 117, 1.1, and 12.3, respectively.

\begin{tabular}{|l|l|l|l|}
\hline \multicolumn{2}{|l|}{} & Mean \pm SD & Median [25\%-75\%] \\
\hline \multirow{5}{*}{ Subjects } & & & \\
& Number & 68 & 68 \\
& Male/female & $40 / 28$ & $40 / 28$ \\
& Age (years old) & $62.1 \pm 10.9$ & $65[57-69]$ \\
& & & \\
\hline \multirow{5}{*}{ Glucose Profile } & Fasting glucose (mg/dL) & $163 \pm 51.9$ & $155[116-207]$ \\
& Average glucose (mg/dL) & $210 \pm 72.0$ & $197[145-265]$ \\
& HbA1c (\%) & $7.9 \pm 1.7$ & $7.8[6.6-9.0]$ \\
& Morbus Value & $215 \pm 235$ & $117[35-344]$ \\
\hline \multirow{5}{*}{ Lipid Profile } & HDL-C (mg/dL) & $68.5 \pm 20.7$ & $63.5[53.0-81.3]$ \\
& LDL-C (mg/dL) & $133 \pm 37.2$ & $136[105-157]$ \\
& Triglyceride (mg/dL) & $127 \pm 109$ & $87.0[63-161]$ \\
& LDL/HDL & $2.0 \pm 0.76$ & $1.8[1.4-2.5]$ \\
\hline \multirow{2}{*}{ Insulin } & HOMA-R & $1.2 \pm 0.7$ & $1.1[0.7-1.6]$ \\
& HOMA- $\beta$ & $15.1 \pm 9.6$ & $12.3[9.1-18.5]$ \\
\hline
\end{tabular}

Table 1: Subjects and fundamental data.

Responses of Glucose and insulin for Carbo70 were shown (Table 2). Increment of glucose / IRI was $48.0 \mathrm{mg} / \mathrm{dL} / 8.6$ $\mu \mathrm{U} / \mathrm{mL}$, respectively. By the comparison of 2 ways of calculation, Delta Ratio of IGI for Carbo70 and AUC ratio of IGI for Carbo70 were 0.14[0.08-0.26] and 3.3[2.5-5.2], respectively.

\begin{tabular}{|c|c|c|c|}
\hline & & Mean \pm SD & Median [25\%-75\%] \\
\hline $\begin{array}{l}\text { Response of } \\
\text { Glucose }\end{array}$ & $\begin{array}{l}\text { Before }(0 \mathrm{~min}) \\
\text { After }(30 \text { mins }) \\
\text { Increment }(0-30)\end{array}$ & $\begin{array}{l}163 \pm 51.9 \\
211 \pm 61.2 \\
48.0 \pm 29.2\end{array}$ & $\begin{array}{l}155[116-207] \\
204[159-270] \\
44[29-61]\end{array}$ \\
\hline $\begin{array}{l}\text { Response of } \\
\text { Insulin }\end{array}$ & $\begin{array}{l}\text { Before }(0 \mathrm{~min}) \\
\text { After }(30 \text { mins }) \\
\text { Increment }(0-30)\end{array}$ & $\begin{array}{l}3.1 \pm 1.1 \\
11.8 \pm 9.1 \\
8.6 \pm 8.7\end{array}$ & $\begin{array}{l}3.2[24-4.1] \\
9.4[6.5-12.4] \\
6.0[3.9-9.5]\end{array}$ \\
\hline $\begin{array}{l}\text { Ratio of } \\
\text { IRI/glucose }\end{array}$ & $\begin{array}{l}\text { Delta (increment) } \\
\text { AUC (square) }\end{array}$ & $\begin{array}{l}0.26 \pm 0.30 \\
4.3 \pm 3.2\end{array}$ & $\begin{array}{l}0.14[0.08-0.26] \\
3.3[2.5-5.2]\end{array}$ \\
\hline
\end{tabular}

Table 2: Response of glucose and IRI for carbo70. 


\section{Comparison among 4 groups}

According to the average blood glucose in a day, subjects were divided into 4 groups. The value of average glucose was 132 [127-138] mg/dL, 170 [159-178] mg/dL, 226 [214-248] mg/dL, 297 [293-333] mg/dL in group 1,2,3,4, respectively. Each group as 17 cases.

Responses of glucose for Carbo70 in 4 groups were shown (Figure 1a). The increment from 0 to 30 mins was 37 $\mathrm{mg} / \mathrm{dL}, 39 \mathrm{mg} / \mathrm{dL}, 45 \mathrm{mg} / \mathrm{dL}, 46 \mathrm{mg} / \mathrm{dL}$ in group 1-4, respectively. Responses of IRI for Carbo70 in 4 groups were shown (Figure 1b). The increment from 0 to 30 mins was $7.5 \mu \mathrm{U} / \mathrm{mL}, 5.6 \mu \mathrm{U} / \mathrm{mL}, 6.1 \mu \mathrm{U} / \mathrm{mL}, 4.2 \mu \mathrm{U} / \mathrm{mL}$ in group 14 , respectively.
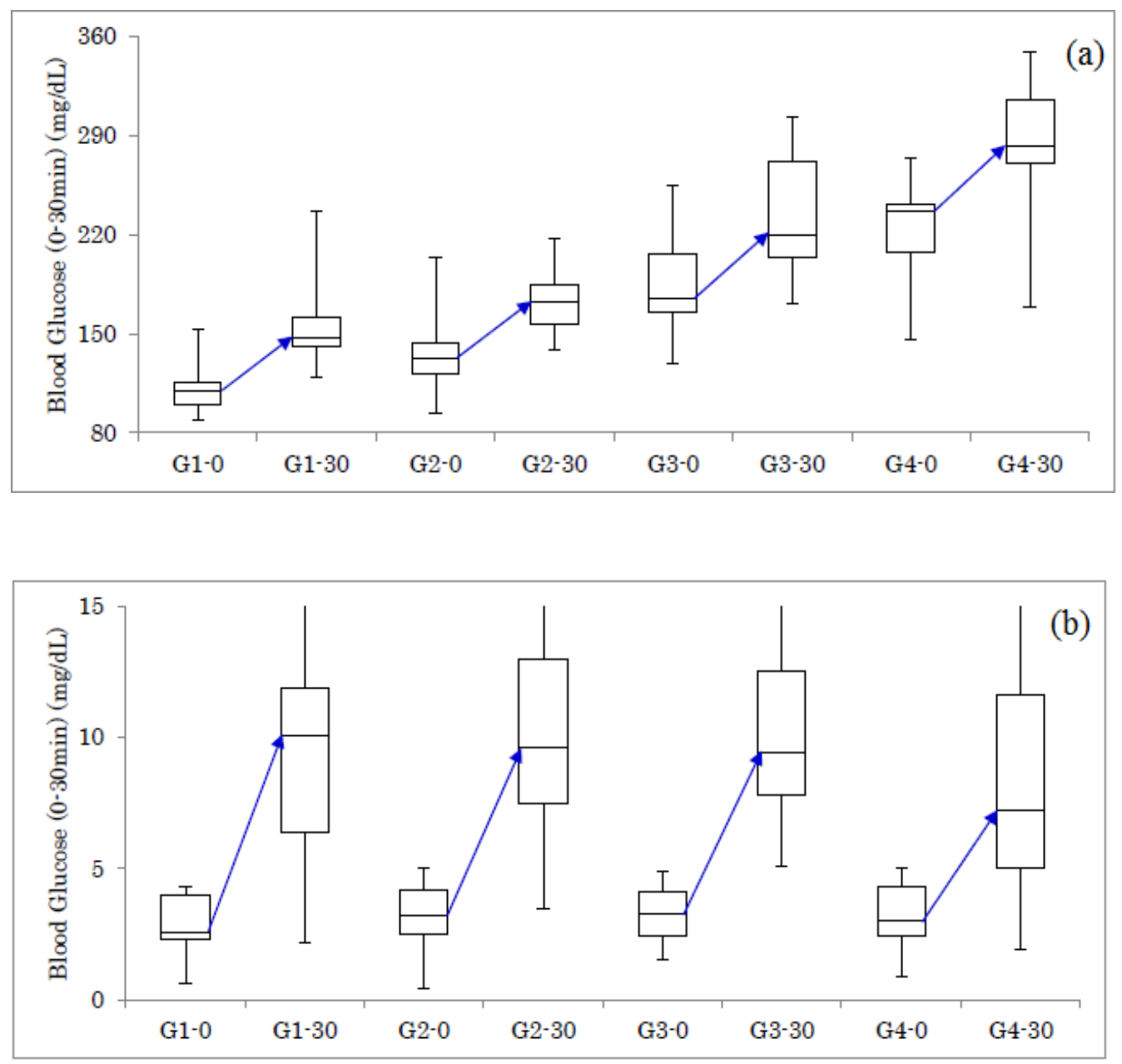

Figure1: Responses of glucose and insulin for Carbo70 in 4 groups. (a) Response of glucose in 4 groups. (b) Response of IRI in 4 groups.

\section{HbA1c and $M$ value}

The HbA1c value in each group was shown (Figure 2a). Median HbA1c was 6.3\%, 6.8\%, 8.5\%, 9.2\%, in group 1-4, respectively. $M$ value in each group was shown (Figure 2b). Median M value was 15, 58, 192, 486, in group 1-4, respectively. In both figures, the obtained data increased in group 1 to 4 . 


\section{Delta Ratio vs AUC Ratio}

Delta ratio of IGI for Carbo70 in each group was shown (Figure 3a). Median value of Delta ratio was 0.14, 0.22, 0.17, 0.10, in group 1-4, respectively. Similarly, the AUC ratio of IGI for Carbo70 in each group was shown (Figure 3b). The median value of AUC ratio was 4.3, 4.6, 3.1, 2.1 in group 1-4, respectively.

When compared the groups between groups $1+2$ and groups $3+4$, the former showed overlapped distribution, while the latter showed divided distribution.
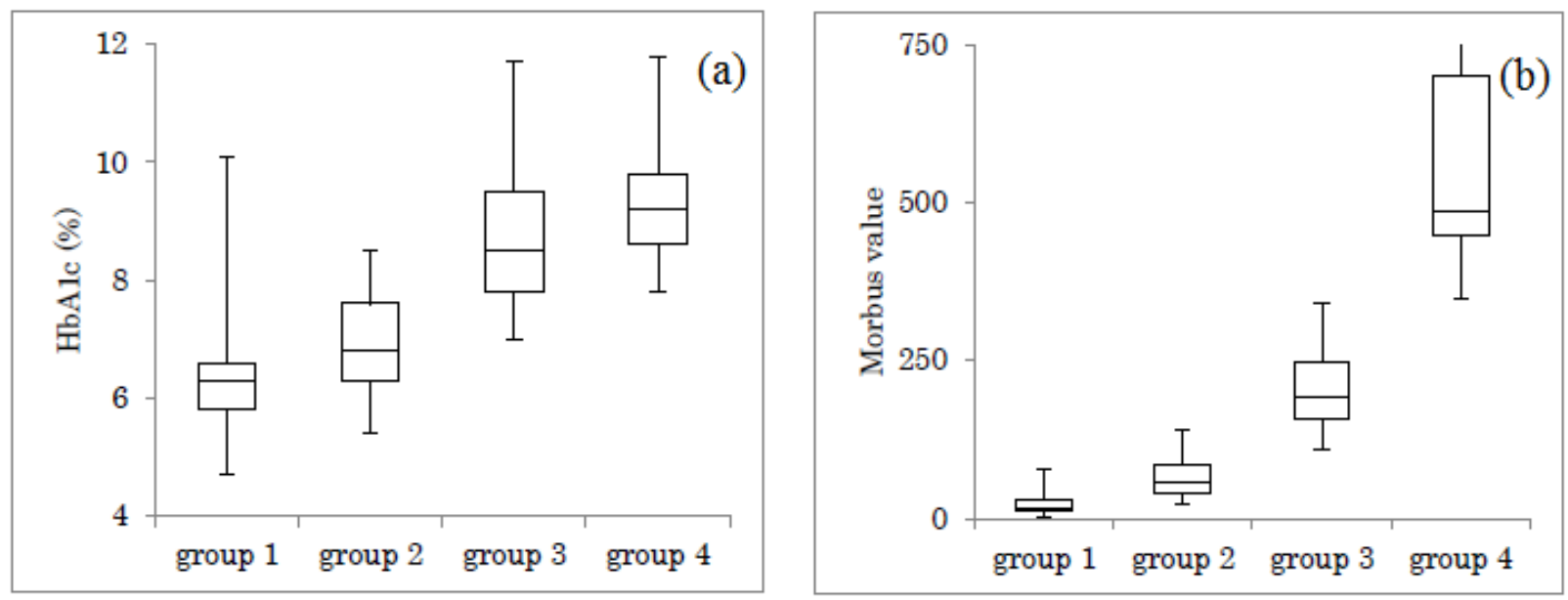

Figure 2: Comparison of HbA1c and $\mathrm{M}$ value in 4 groups. (a) The level of HbA1c in 4 groups. (b) The level of M value in 4 groups.
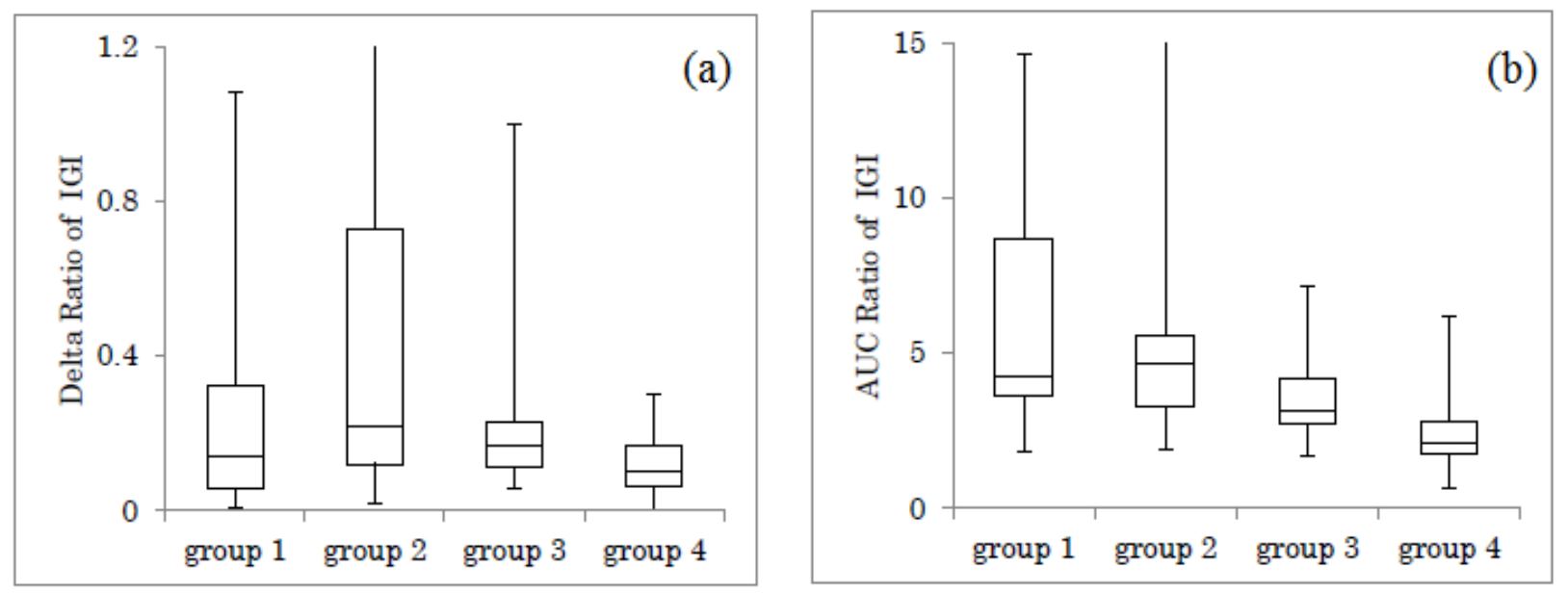

Figure 3: Comparison of IGI ratio between Delta and AUC in 4 groups. (a) Delta Ratio of IGI for Carbo70. (b) AUC Ratio of IGI for Carbo70.

\section{Correlation among several biomarkers}

There was a significant correlation between average glucose and HbA1c $(\mathrm{p}<0.01)$ (Figure 4a) and a significant correlation between average glucose and $M$ value $(p<0.01)$ (Figure $4 b)$.

In comparison with Delta ratio and AUC ratio, both correlations to average blood glucose were shown (Figure 5a and $5 b)$. Both revealed significant correlations, and the latter showed a higher correlation coefficient than the former (Figure 5a and 5b). 

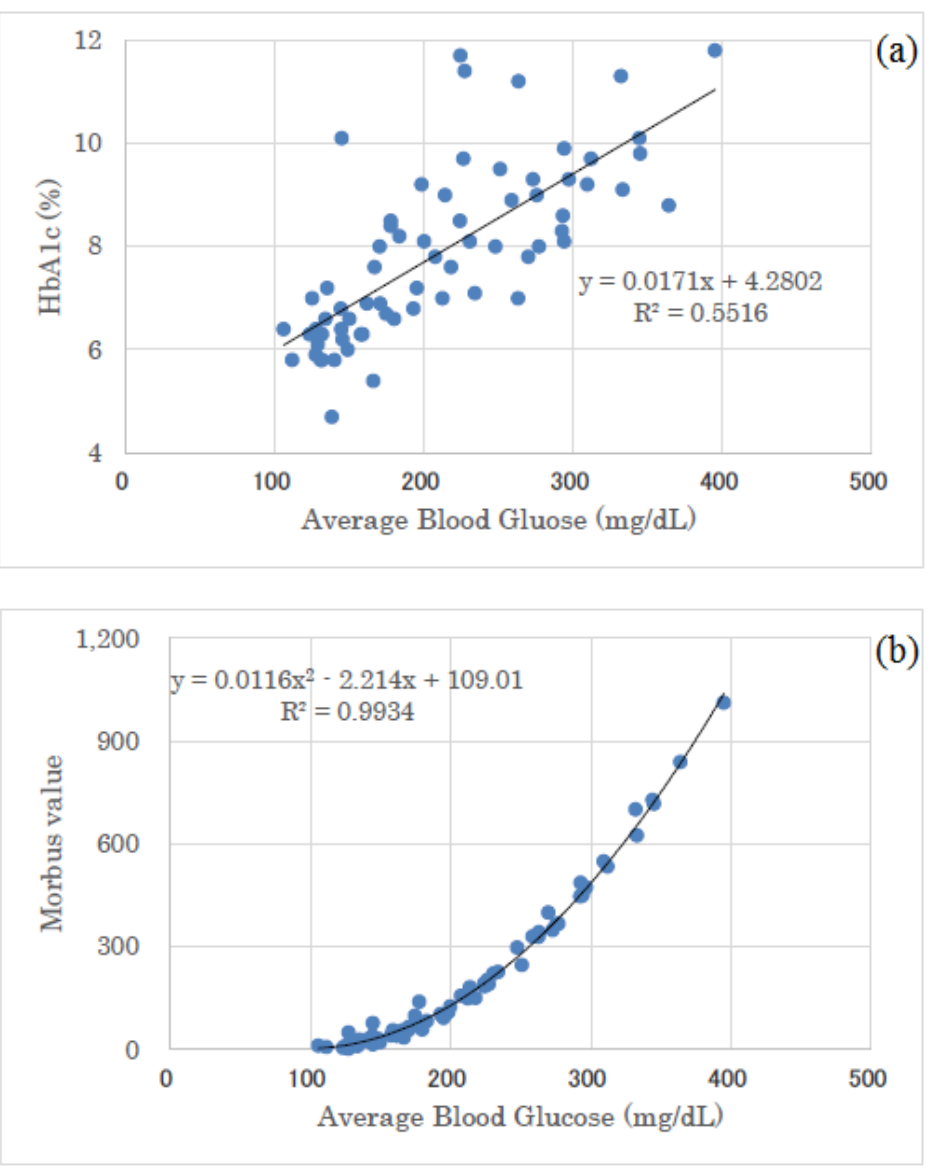

Figure 4: Correlation between average glucose and HbA1c and $\mathrm{M}$ value. (a) There was a significant correlation between average glucose and HbA1c $(\mathrm{p}<0.01)$. (b) There was a significant correlation between average glucose and $\mathrm{M}$ value $(\mathrm{p}<0.01)$.

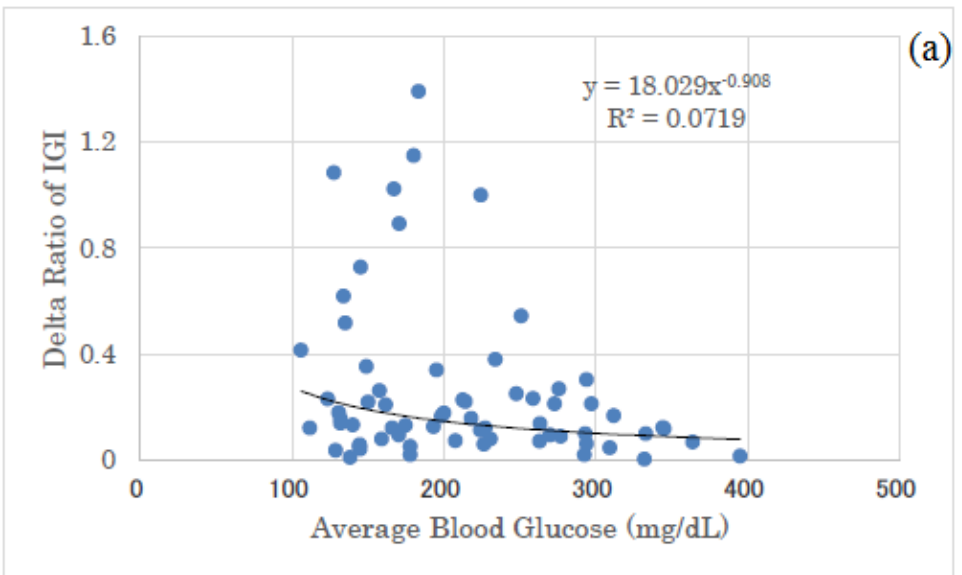




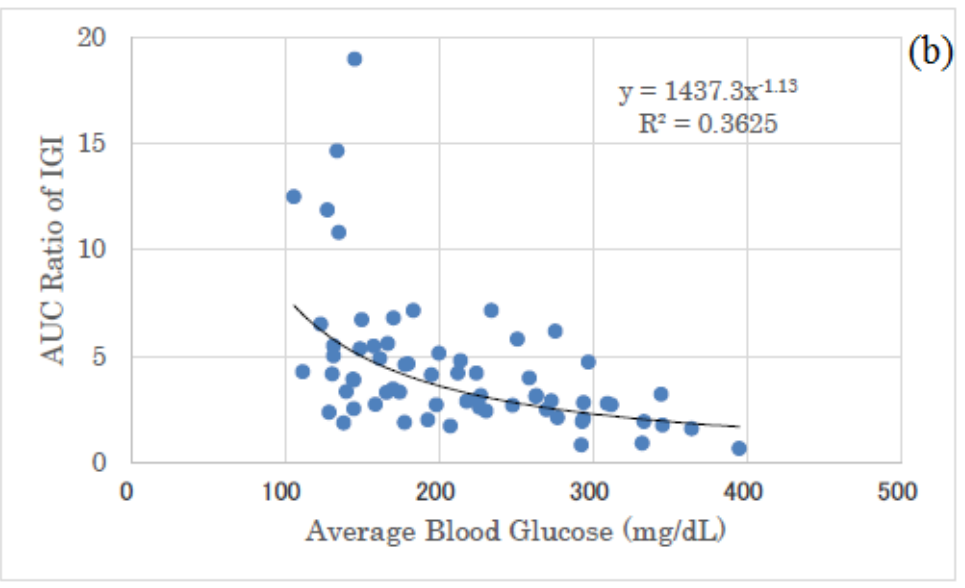

Figure 5: Correlation between average glucose and Delta/AUC ratio of IGI. (a) There was significant correlation between average glucose and Delta ratio of IGI for Carbo70 ( $\mathrm{p}<0.01)$. (b) There was significant correlation between average glucose and AUC ratio of IGI for Carbo70 $(\mathrm{p}<0.01)$.

\section{Discussion}

In the current study, subjects were 68 cases which were divided into 4 groups according to mean blood glucose level. There were the response of IRI and glucose for the loading of $70 \mathrm{~g}$ carbohydrate. As an average glucose increase from group 1 to 4, there was the tendency of increasing increment of glucose and decreasing increment of insulin. These results may be related to a decrease in insulin secretion due to glucose toxicity from persisting hyperglycemia for long.

In the group 1-4, the distribution of HbA1c showed a wide overlap, but the $\mathrm{M}$ value has little overlap with a large difference in the numerical value. The reason for the large difference in $\mathrm{M}$ value is due to the fact that both average value and variation of glucose are quantified. Consequently, it seems to be useful to estimate by one numerical value for two factors.

Comparison of Delta Ratio and AUC Ratio in the four groups revealed that the difference in the 4 groups was not so clear in the former, but the median value in the latter showed a downward trend in the group from 1 to 4 . One reason for this may be due to larger variation and distribution in the former.

As to the correlation between average glucose and HbA1c, the linear regression shows that $y=0.0171 \mathrm{x}+4.28$. When $\mathrm{x}$-axis and $\mathrm{y}$-axis are converted, the formula became that $\mathrm{y}=32.2 \mathrm{x}-43.6$.

There was a well-known correlation between the both by Nathan et al. [29]. Its formula was that blood glucose $(\mathrm{mg} / \mathrm{dL})=28.7 \mathrm{x} \mathrm{HbA1c}(\%)-46.7$. It was calculated from approximately 2700 glucose samples, and it did not differ significantly in the subgroups on age, sex, diabetes type, race, ethnicity or smoking status [29].

Consequently, the linear regression equations are similar between ours and Nathan, suggesting that our correlation between average blood glucose and $\mathrm{HbA1c}$ would be reliable.

There was a significant correlation between the mean blood glucose level and the $\mathrm{M}$ value, and it was expressed by a quadratic equation of $y=0.0116 x^{2}-2.214 x+109.01$. This is because the calculation of the $\mathrm{M}$ value is a quadratic equation, from the definition of $\mathrm{M}$ value [25-27]. By comparing the correlation between mean blood glucose and delta IGI, the correlation between mean blood glucose and AUC-IGI, the latter correlation coefficient was higher.

The reason for this would be from the results that Delta ratio has larger numerical variations and AUC ratio has smaller variations. From these difference, both research methods are useful for investigation for clinical research, but AUC ratio seems to be a little superior to Delta ratio because of less overlap and somewhat divided distribution of the data in a grouped situation. 
Our current study is to examine pancreas function by loading carbohydrates. Conventionally, intravenous glucose tolerance test (IVGTT) and OGTT have been used [30,31]. In recent years, a method of a meal tolerance test (MTT) has been rather prevalent.

For MMT, several reports were found in the clinical study for breakfast. One is a standardized mixed meal tolerance test (MMTT) for assessment of the ability of beta cell of the pancreas [32].

Another MTT study revealed that the protocol is breakfast with carbohydrate 55\% and $500 \mathrm{kcal}$. The amount of carbohydrate is $69 \mathrm{~g}$, and this is almost the same as that of our study [33]. According to the data for T2DM, the response of glucose and IRI from 0 to 30 mins seemed to be 167 to $230 \mathrm{mg} / \mathrm{dL}, 12$ to $37 \mu \mathrm{U} / \mathrm{mL}$, respectively. When calculated from these results, Delta ratio of IGI and AUC ratio of IGI is 0.39 and 12.3, respectively. These levels are compatible for those of group 1 and 2.

There was another paper with 2 types of formula breakfast provided. Carbo-group took breakfast with $\mathrm{PFC}=15: 20: 65 \%$, and protein-group took breakfast with $\mathrm{PFC}=35: 20: 45 \%$ [34]. In response to the same lunch in both groups, protein-group revealed higher IRI secretion and lower glucose increase, indicating the second-meal phenomenon with stable glucose variability.

After the intake and ingestion of MTT, there was GLP-1-induced insulin secretion found [35]. By the preload of a mixed meal, glucose tolerance was relieved due to the degree of T2DM [36]. Consequently, to investigate the influence of macronutrients would explore the mechanism of T2DM, especially the glucose variability.

The current study has some limitation for the method and speculation. There have been lots of studies about MTT with a variety of content or a complex ratio of macronutrients. Then, the condition of digestion, absorption, insulin secretion, and glucose response can vary. However, our results in this study would be the basal data for the developing clinical diabetic research in the future.

In relation to the current study, a discussion has been continued about the efficacy of LCD and CR on diabetes in the world. Authors and colleagues have continued various research into two aspects related to this field. One is the research for glucose variability, $\mathrm{M}$ value, urinary $\mathrm{C}$-peptide and so on, associated with several correlations among biomarkers [25, 26]. Another aspect includes the MTT. We have used breakfast including 70g of carbohydrate, which is the standard CR formula by Japan Diabetes Association (JDA) [37,38]. The method includes the responses of glucose, insulin, and C-peptide, which is similar to 75gOGTT [21,39]. In the clinical setting, MTT has been applied for the evaluation of insulin secretion $[33,40]$.

The current study is the joint research of the two axes mentioned above. $M$ value indicates both average blood glucose level and mean amplitude of glycemic excursions (MAGE) [23-26]. Then, glucose variability for a day is described. In addition, research of Delta or AUC ratio of IGI supposes the pancreatic function of meal loading for 30 mins. Therefore, various pathophysiological conditions related to diabetes have been investigated.

Obtained data in $\mathrm{M}$ value was wider distribution than that of HbA1c. This is a beneficial point for clinical research in the case of clinical study for correlation and difference, with the less overlapped area.

As mentioned above, we discussed the speculation of current data, previous references of MTT and clinical research about CR/LCD.

There is a limitation of this research concerning MTT. In a mixed meal test, the degree of digestion and absorption may vary in a different protocol of the research. However, this study would be one of the basal reference data in the light of developing research of MTT.

\section{Conclusion}

In summary, T2DM patients were investigated for daily glucose profile, average glucose and $\mathrm{M}$ value. In addition, IGI of insulin/glucose (0-30 mins) for Carbo70 was calculated and compared by tow way, Delta Ratio of IGI for Carbo70 and AUC Ratio of IGI for Carbo70. Obtained these results suggest that obtained data would be reference data in MTT in the future study. 


\section{Conflicts of Interest}

The authors declare that they have no conflicts of interest.

\section{Acknowledgments}

As regard to the current article, some part was presented in the Annual Congress of Japan Diabetes Society (JDS) 90th, Tokyo, 2018. The authors express their gratitude to all staff and patients for their understanding and cooperation.

\section{References}

1. Reaven GM. Role of insulin resistance in human disease. Diabetes. 1988;37(12):1595-607.

2. Hall ME, Wang Z, Carmo JD, et al. Obesity and Metabolic Syndrome Hypertension. In: Berbari A, Mancia G, eds. Disorders of Blood Pressure Regulation. Cham: Springer; 2018. p. 705-22.

3. Mendonça FM, de Sousa FR, Barbosa AL, et al. Metabolic syndrome and risk of cancer: Which link? Metabolism. 2015;64(2):182-89.

4. Ojo O. An overview of diabetes and its complications. Diabetes Res Open J. 2016;2(2): e4-e6.

5. American Diabetes Association. Improving Care and Promoting Health in Populations: Standards of Medical Care in Diabetes-2019. Diabetes Care. 2019;42(Supplement 1):S7-S12.

6. American College of Physicians. Clinical Guidelines and Recommendations. 2017.

7. American Diabetes Association. Pharmacologic Approaches to Glycemic Treatment: Standards of Medical Care in Diabetes-2018. Diabetes Care. 2018;41(Supplement 1):S73-S85.

8. International Diabetes Federation. Standards of Medical Care in Diabetes-2015. Diabetes Care. 2015;38 Suppl 1:S1-S2.

9. Gardner CD, Trepanowski JF, Del Gobbo LC, et al. Effect of Low-Fat vs Low-Carbohydrate Diet on 12Month Weight Loss in Overweight Adults and the Association With Genotype Pattern or Insulin Secretion The DIETFITS Randomized Clinical Trial. JAMA. 2018;319(7):667-79.

10. Tay J, Thompson CH, Luscombe-Marsh ND, et al. Effects of an energy-restricted low carbohydrate, high unsaturated fat/low saturated fat diet versus a high-carbohydrate, low-fat diet in type 2 diabetes: A 2-year randomized clinical trial. Diabetes, Obes Metab. 2017;20(4):858-71.

11. Feinman RD, Pogozelski WK, Astrup A, et al. Dietary carbohydrate restriction as the first approach in diabetes management: Critical review and evidence base. Nutrition. 2015;31(1):1-13.

12. Atallah R, Filion KB, Wakil SM, et al. Long-Term Effects of 4 Popular Diets on Weight Loss and Cardiovascular Risk Factors: A Systematic Review of Randomized Controlled Trials. Circ Cardiovasc Qual Outcomes. 2014;7(4):815-27.

13. Miller V, Yusuf S, Chow CK, et al. Availability, affordability, and consumption of fruits and vegetables in 18 countries across income levels: findings from the Prospective Urban Rural Epidemiology (PURE) study. Lancet Glob Health. 2016;4(10):e695-e703.

14. Dehghan M, Mente A, Zhang X, et al. Associations of fats and carbohydrate intake with cardiovascular disease and mortality in 18 countries from five continents (PURE): a prospective cohort study. Lancet. 2017;390(10107):2050-62. 
15. Ogurtsova K, da Rocha Fernandes JD, Huang Y, et al. IDF Diabetes Atlas: Global estimates for the prevalence of diabetes for 2015 and 2040. Diabetes Res Clin Pract. 2017;128(1):40-50.

16. Bernstein RK. Dr. Bernstein's Diabetes solution: The complete guide to achieving normal blood sugars. 4th ed. New York: Little, Brown and Company; 2007.

17. Bando H, Ebe K, Nakamura T, et al. Low Carbohydrate Diet (LCD): Long and short-term effects and hyperketonemia. Glycative Stress Research. 2016;3(4):193-204.

18. Muneta T, Kawaguchi E, Nagai Y, et al. Ketone body elevation in placenta, umbilical cord, newborn and mother in normal delivery. Glycative Stress Research. 2016;3(3):133-40.

19. Bando H, Ebe K, Muneta T, et al. Investigation of uric acid and cystatin C on low-carbohydrate diet (LCD). Diabetes Res Open J. 2017;3(2):31-8.

20. Ebe K, Bando H, Muneta T, et al. Effect of low carbohydrate diet (LCD) for diabetic patients with hypertriglycemia. Endocrinol Metab. 2017;1(4):1-9.

21. Bando H, Ebe K, Muneta T, et al. Proposal for Insulinogenic Index (IGI)-Carbo70 as Experimental Evaluation for Diabetes . J Clin Exp Endocrinol. 2017;1(1):1-5.

22. Japan Diabetes Association. Diabetes clinical practice guidelines based on scientific evidence. 2013.

23. Moberg E, Kollind M, Lins PE, et al. Estimation of blood-glucose variability in patients with insulindependent diabetes mellitus. Scand J Clin Lab Invest. 1993;53(5):507-14.

24. Kusunoki Y, Katsuno T, Nakae R, et al. Evaluation of blood glucose fluctuation in Japanese patients with type 1 diabetes mellitus by self-monitoring of blood glucose and continuous glucose monitoring. Diabetes Res Clin Pract. 2015;108(2):342-49.

25. Schlichtkrull J, Munck O, Jersild M. The M-value, an index of blood sugar control in diabetics. J Intern Med. 1965;177(1):95-102.

26. Service FJ, Molnar GD, Rosevear JW, et al. Mean amplitude of glycemic excursions, a measure of diabetic instability. Diabetes. 1970;19(9):644-55.

27. Siegelaar SE, Holleman F, Hoekstra JBL. et al. Glucose Variability; Does It Matter? Endocr Rev. 2010;31(2):171-82.

28. Yanai H. Four step excel statistics. $4^{\text {th }}$ ed. Tokyo: Seiun-sha Publishing Co. Ltd.; 2015.

29. Nathan DM, Kuenen J, Borg R, et al. Translating the A1C Assay Into Estimated Average Glucose Values. Diabetes Care. 2008;31(8):1473-78.

30. Cersosimo E, Solis-Herrera C, Trautmann ME, et al. Assessment of pancreatic $\beta$-cell function: review of methods and clinical applications. Curr Diabetes Rev. 2014;10(1):2-42.

31. Veijola R, Koskinen M, Helminen O, et al. Dysregulation of glucose metabolism in preclinical type 1 diabetes. Pediatr Diabetes. 2016;17(S22):25-30.

32. Shankar SS, Lee DS, Raymond RH, et al. Outpatient versus inpatient mixed meal tolerance and arginine stimulation testing yields comparable measures of variability for assessment of beta cell function. Contemp Clin Trials Commun. 2018;10:94-99.

33. Cozma LS, Luzio SD, Dunseath GJ, et al. Beta-cell response during a meal test: a comparative study of incremental doses of repaglinide in type 2 diabetic patients. Diabetes Care. 2005;28(5):1001-07. 
34. Park YM, Heden TD, Liu Y, et al. A high-protein breakfast induces greater insulin and glucose-dependent insulinotropic peptide responses to a subsequent lunch meal in individuals with type 2 diabetes. J Nutr. 2015;145(3):452-58.

35. Man CD, Micheletto F, Sathananthan M, et al. Model-Based Quantification of Glucagon-Like Peptide-1Induced Potentiation of Insulin Secretion in Response to a Mixed Meal Challenge. Diabetes Technol Ther. 2016;18(1):39-46.

36. Tricò D, Baldi S, Tulipani A, et al. Mechanisms through which a small protein and lipid preload improves glucose tolerance. Diabetologia. 2015;58(11):2503-12.

37. Bando H, Ebe K, Muneta T, et al. Urinary C-Peptide Excretion for Diabetic Treatment in Low Carbohydrate Diet (LCD). Journal of Obesity and Diabetes. 2018;1(1):13-18.

38. Monnier L, Colette C, Owens DR. Glycemic variability: The third component of the dysglycemia in diabetes. Is it important? How to measure it? J Diabetes Sci Technol. 2008;2(6):1094-100.

39. Forbes S, Lam A, Koh A, et al. Comparison of metabolic responses to the mixed meal tolerance test vs the oral glucose tolerance test after successful clinical islet transplantation. Clin Transplant. 2018;32(8):e13301.

40. Ohkura T, Shiochi H, Fujioka Y, et al. 20/(fasting C-peptide $\times$ fasting plasma glucose) is a simple and effective index of insulin resistance in patients with type 2 diabetes mellitus: a preliminary report. Cardiovasc Diabetol. 2013;12:21. 